\title{
Chinese expert brief consensus on newborn screening of inherited metabolic disorders during the novel coronavirus infection epidemic
}

\author{
Shan Liu ${ }^{1}$, Dongjuan Wang ${ }^{1}$, Kexing Wan ${ }^{1}$, Hao Liu ${ }^{1}$, Dayong Zhang ${ }^{1}$, Jing Yang ${ }^{1}$, Zhaojian Yuan ${ }^{1}$, \\ Xiaoyan $\mathrm{He}^{1}$, Jie Wang ${ }^{2}$, Mingcai Ou ${ }^{3}$, Wenbin $\mathrm{Zhu}^{4}$, Yiping $\mathrm{Qu}^{5}$, Dehua Zhao ${ }^{6}$, Weipeng Wang ${ }^{7}$, Shiguo Liu ${ }^{8}$, \\ Maosheng Gu', Shuodan Huang ${ }^{10}$, Zhiguo Wang ${ }^{11}$, Lin Zou ${ }^{1}$ \\ ${ }^{1}$ Newborn Screening Center/Center for Clinical Molecular Laboratory Medicine, National Clinical Research Center for Child Health and \\ Disorders, Ministry of Education Key Laboratory of Child Development and Disorders, China International Science and Technology Cooperation \\ Base of Child Development and Critical Disorders, Children's Hospital of Chongqing Medical University, Chongqing 400014, China; ${ }^{2}$ Center \\ of Clinical Laboratory, Maternal and Child Health Care Hospital of Hainan Province, Haikou 570000, China; ${ }^{3}$ Newborn Screening Center of \\ Sichuan, Maternal and Child Health Hospital of Sichuan Province, Chengdu 610000, China; ${ }^{4}$ Fujian Neonatal Screening Center, Fujian Maternal \\ and Children Health Hospital, Fuzhou 350000, China; ${ }^{5}$ Newborn Screening Center of Zhejiang, Children's Hospital of Zhejiang University School \\ of Medicine, Hangzhou 310000, China; ${ }^{6}$ Newborn Screening Center of Henan Maternal and Child Health Hospital, the Third Affiliated Hospital \\ of Zhengzhou University, Zhengzhou 450000, China; ${ }^{7}$ Health Care Department, Maternal and Child Health Hospital of Hubei Province, Wuhan \\ 430000, China; ${ }^{8}$ Qingdao Women \& Children Hospital Neonatal Screening Lab, Qingdao 266000, China; ${ }^{9}$ Genetic Center of Xuzhou Maternal and \\ Children Health Hospital, Xuzhou 221000, China; ${ }^{10}$ Meizhou Women \& Children Hospital, Meizhou 514000, China; ${ }^{11}$ National Center for Clinical \\ Laboratories, Beijing Hospital, National Center for Gerontology, Institute of Geriatric Medicine, Chinese Academy of Medical Sciences, Beijing \\ 100000, China \\ Correspondence to: Lin Zou. Newborn Screening Center/Center for Clinical Molecular Laboratory Medicine, Children's Hospital of Chongqing \\ Medical University, 136 Zhongshan Er Road, Yuzhong District, Chongqing 400014, China. Email: zoulin74@126.com.
}

\begin{abstract}
Novel coronavirus (2019-nCov) infection (COVID-19) rapidly spread across China and 25 countries in the worldwide, which infected not only adults but also children, even neonates. Each year, about 15 million newborns are delivered in China. Newborn screening (NBS) helps effectively prevent some mental retardation, premature death, and adverse outcomes in the early stage of baby, which could detect some inherited metabolic disorders (IMDs). During this COVID-19 epidemic, how to balance the risk of infected 2019-nCov and the risk of disability and teratogenesis of IMDs. Expert members of NBS extra quality assessment in National Clinical Center of Laboratory (NCCL) give a brief consensus for NBS of IMDs in the COVID-2019 epidemic, hoping that the brief consensus could be reference for NBS of IMDs in the other epidemic areas or periods all over the world.
\end{abstract}

Keywords: Novel coronavirus (2019-nCov); newborn screening (NBS); inherited metabolic disorders (IMDs)

Submitted Feb 21, 2020. Accepted for publication Mar 27, 2020.

doi: $10.21037 /$ atm.2020.03.60

View this article at: http://dx.doi.org/10.21037/atm.2020.03.60

Since December 2019, a sustained increasing number of cases with novel coronavirus (2019-nCov) infection (COVID-19) originated in Wuhan has been identified, rapidly spread across China and 25 countries in the worldwide. By Feb 25, more than 77,000 cases had been diagnosed as COVID-19 in China, and the mortality was about $3.42 \%$. Only adults are susceptible to $2019-\mathrm{nCov}$ infection in early reports $(1,2)$. But, more children (3) with COVID-19 were diagnosed recently, the youngest being $30 \mathrm{~h}$ after birth (4). The children's health and medical care is raised great attention during the epidemic of COVID-2019.

Neonates are thought to be susceptible to the virus 
because their immune system is not well developed. The newborn screening (NBS) for inherited metabolic disorders (IMDs) helps effectively prevent some mental retardation, premature death, and adverse outcomes in the early stage of a baby, which could detect some treatable IMDs, including congenital hypothyroidism $(\mathrm{CH})$, hyperphenylalaninemia (HPA), congenital adrenocortical hyperplasia (CAH), glucose-6-phosphatase (G6PD) deficiency, methylmalonic academia (MMA), maple syrup urine disease (MSUD), primary carnitine deficiency (PCD), and so on. NBS is one of the effect measures to prevent birth defects, which can screen and diagnose early, treat timely and effectively, to avoid or reduce disability, to improve the quality of children life, and ultimately improve the quality of population. NBS is a highly systematized program with multiple procedures, including informed consent, blood collection, laboratory testing, and complicated post-testing management in the different medical organizations (5), with the whole process of screening, confirmation, diagnosis, treatment, follow-up and management.

Each year, about 15 million newborns are delivered. During this epidemic, there still some newborns are produced and have potential harmful for NBS. The members belonging to the subdivision of NBS extra quality assessment in National Clinical Center of Laboratory (NCCL) have contributed to great efforts for NBS in China. We aim to elicit a contingency consensus for NBS of IMDs in the COVID-2019 epidemic, mainly focused on the whole strategy for NBS.

\section{For screening of NBS for IMDs}

\section{Health education and informed consent}

The medical staff of delivery hospitals, regardless of the 2019-nCoV epidemic area or not, still need to give routine health education for the guardians of neonates about NBS for IMDs, including the disease type, purpose, significance, cost, method, importance of early screening diagnosis/ treatment, and sign the informed consent.

Besides, the medical staff must explain the harmful of COVID-19 in detail to the neonates' guardians. If the parturients and newborns are infected with the 2019$\mathrm{nCoV}$, they are easy to have various complications, such as myocarditis and meningitis, which will cause serious consequences and even endanger life if not treated in time. Meanwhile, the medical staff must detailed enquire neonates' guardians whether their family members closely contacted the diagnosed or suspected COVID-19 cases, any influenza-like symptoms or normal temperature recently, and record them correctly.

\section{Sample collecting and testing}

The sample collection and detection are performed as the routine process of NBS for IMDs during this period. But for the neonates whose mothers are suspected or diagnosed COVID-19 would not be performed routine NBS, until these neonates are released from quarantine after 14-day isolation and clinical observation. And the results of 2019-nCoV's RNA detection from these neonates are negative in twice, they are permitted to collect dried blood spots (DBS) for routine NBS. Only when the neonates have clear family history of IMDs, exhibit clinical manifestation of IMDs with life crisis at the early stage, the NBS for IMDs should be performed and transferred to the superior hospitals immediately. And the subsequent procedure will strictly follow the prevention and control law of infectious diseases of China (6). The medical staff shall fully perform personal biosafety protection (wearing medical protective clothing and gloves, masks, goggles, etc.) (7-9), collect specimen from heel blood (or using other tests' venous blood for DBS), put it in a special closed and isolated area (e.g., biosafety cabinet) for drying them naturally, seal and keep them with "one sample one storage bag" with biosafety hazard signs, and inform NBS center with enough biosafety protection conditions to test the single specimen independently. After the tests, the storage area for sample must be thoroughly disinfected by ultraviolet irradiation or disinfectants later (7-9).

For the newborns suspected or diagnosed COVID-19 should be postpone the sample collection time till they are excluded from the 2019-nCoV infection, and perform the routine NBS. The medical staff of laboratory should protect themselves in the corresponding biosafety level and test the 2019-nCoV RNA according to SOP recommended by the National Health Committee (7-9).

After test samples from suspected or diagnosed COVID-19 neonates, the specimens were still sealed in accordance with the "one sample one bag" or directly discarded as medical waste referred to "The biological safety guidelines for 2019-nCoV laboratory" (2nd Edition) (8). After finishing the test, the NBS laboratory should be disinfected thoroughly in the air, floor, table and ground according to the requirements (7-9). 
Table 1 Diagnosis and treatment suggestion for common IMDs during COVID-19 epidemic

\begin{tabular}{|c|c|c|}
\hline IMD name & Major abnormal tests & Suggestion \\
\hline $\mathrm{CAH}$ & $17-\mathrm{OHP} \uparrow$ & $\begin{array}{l}\text { Without abnormal symptoms and } 17-\mathrm{OHP} \text { value }<100 \mathrm{nmol} / \mathrm{L} \text {, put off the visiting time of NBS } \\
\text { for confirmation. But with manifestations, e.g., poor response, feeding difficulties, malignant } \\
\text { vomiting, diarrhea., go to the hospital for diagnosis. If } 17-\mathrm{OHP} \text { value }>100 \mathrm{nmol} / \mathrm{L} \text { or with } \\
\text { obvious symptoms, go to NBS center immediately and start treatment asap }\end{array}$ \\
\hline $\begin{array}{l}\text { Non-confirmed } \\
\text { IMDs }\end{array}$ & $\begin{array}{l}\text { Screened positive } \\
\text { results but not IMDs } \\
\text { diagnosis }\end{array}$ & $\begin{array}{l}\text { Contact the specialist in NBS center in time with abnormal manifestations. Confirmed IMDs, } \\
\text { immediately start individualized therapy and plans (the local cases be given therapeutic } \\
\text { guidance by telemedicine }\end{array}$ \\
\hline
\end{tabular}

$\uparrow$, adding. NBS, newborn screening; DBS, dried bloodspots; $\mathrm{CH}$, congenital hypothyroidism; HPA, hyperphenylalaninemia; CAH, congenital adrenocortical hyperplasia; IMDs, inherited metabolic disorders; Phe, phenylalanine; TSH, thyroid stimulating hormone; 17OHP, 17 $\alpha$-hydroxyprogesterone; TMS, tandem mass spectrometry; e.g., for example; asap, as soon as possible.

\section{Reexamination of neonates with positive results in the first screening}

For the newborns with positive IMDs results in the first screening, the medical staff should inform the family (by phone, e-mail, or WeChat public) to collect sample for reexamination in their nearest delivery hospital with full biosafety self-protection.

For the newborns with positive results in the first screening of suspected or diagnosed COVID-19 parturient, we suggest that they should not go to delivery hospital for sample collecting reexamination until the neonates are excluded 2019-nCoV infection. But if the newborns are suspected for G6PD deficiency in the first screening, the family should strictly obey the rules for the G6PD deficiency patients from then, including avoiding some fava beans, medicine and serious infection, lasting the end of the epidemic stage. However, if the neonates have the urgent manifestation of acute hemolysis, they should be taken into the hospital promptly.

\section{For diagnosis and treatment of NBS for IMDs}

The newborns with IMDs positive results of recalling reexamination need further differential diagnosis and confirmation. The IMDs newborns should be treated by IMDs specialist followed the related guidelines (10). During this COVID-19 epidemic, we suggest different treatment for different IMDs as in Table 1.

\section{For follow-up and management of NBS for IMDs}

Most IMDs are chronic diseases, which require longterm medication and regular review. To better control the epidemic, government has adopted closed management to reduce cross-infection caused by personnel flow. For IMDs children, under the condition of guaranteeing medication, the reexamination should be postponed during the epidemic, suggested to keep contact with the medical staff of the NBS center through the telecommunication at any time, to minimize the impact on disease control. For those confirmed $\mathrm{CH}$ or other IMDs, MMA and propionic acidemia, as well as amino acid metabolic diseases such as HPA requiring long-term special food control, they are suggested not stop taking medication or special food.

If those IMDs children have the manifestation of fever, stress, or overeating during the epidemic, they are easy to 


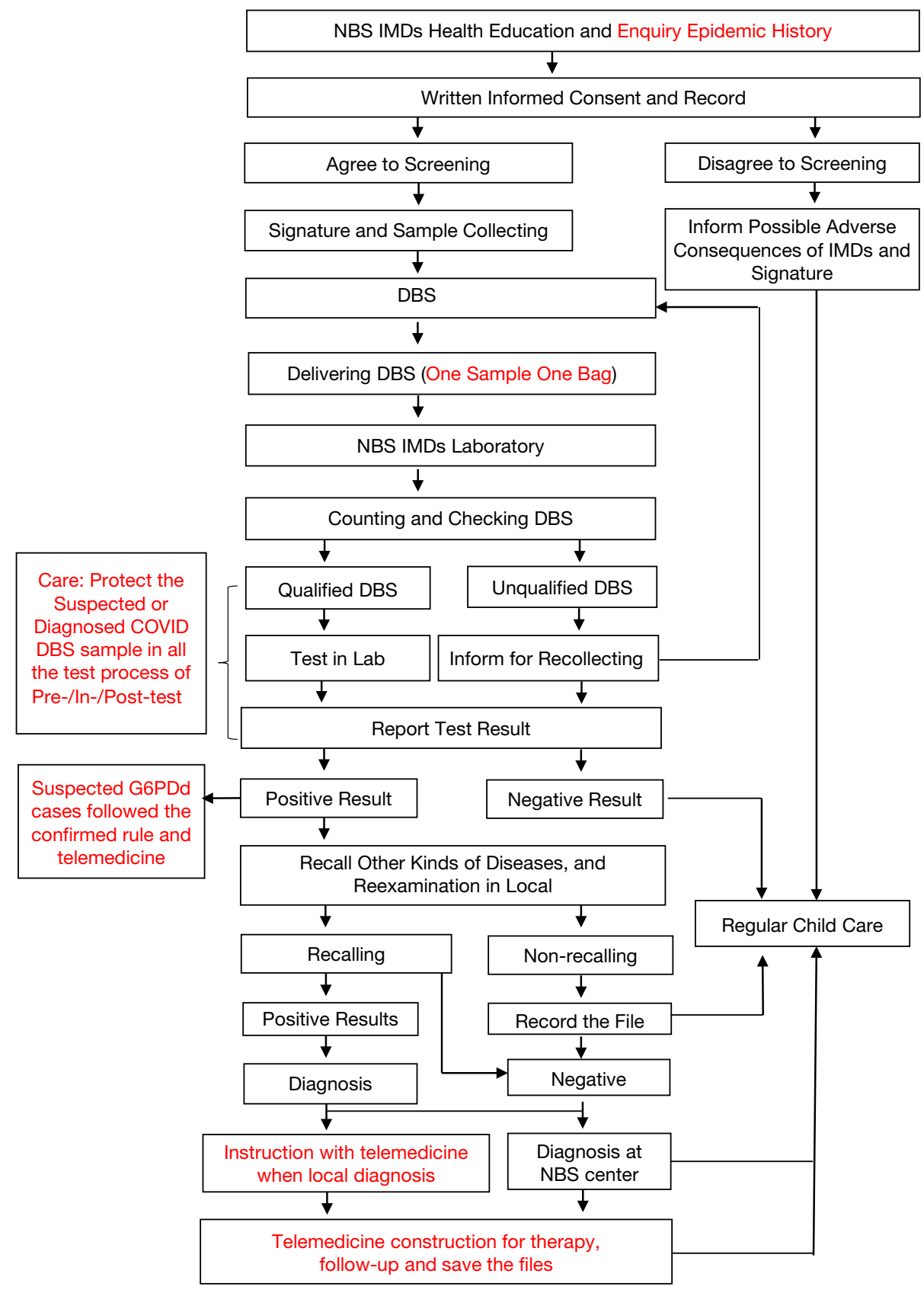

Figure 1 The flow chart for newborn screening of IMDs during the COVID-19 period. The figure shows the flow chart for newborn screening of IMDs. And the difference during COVID-19 is marked in red. NBS, newborn screening; IMDs, inherited metabolic disorders; DBS, dried bloodspots; COVID, novel coronavirus infection disease; Lab, laboratory.

induce acute exacerbation of the disease, and suggested to consult NBS specialist online or remotely guidance for medicine or dietary adjustment. If those IMDs children have poor appetite, poor complexion, frequent vomiting and diarrhea, they are suggested to see a doctor in time with personal biosafety protection.

The recommended flow of NBS for IMDs during the COVID-19 period is shown in Figure 1. 


\section{Suggestion for protection of IMDs children with during epidemic of COVID-19 $(11,12)$}

\section{Self-monitoring}

Considering that infants and young children are unable to effectively express discomfort, the guardians should pay attention to monitoring body temperature, mental appetite, whether with some clinical symptoms such as cough, diarrhea, etc. If neonates have fever, respiratory symptoms, cough, runny nose, vomiting, diarrhea and other gastrointestinal symptoms, they are suggested to consult the specialist of NBS center by telemedicine or see a doctor nearby.

\section{Self-protection}

Considering that health habit is good for prevention infection diseases, the guardians should practice hand hygiene and personal hygiene, maintain respiratory hygiene, wear masks correctly.

\section{Suggestions for diet and bome}

Considering that children with non-special diet need eat more balanced diet, the guardians should give them highquality protein food, fresh vegetables, fruits, enough water, cooked thoroughly, ventilation and cleaning at home, to avoid intimate contact, ensure adequate sleep and improve immunoresistance.

\section{Suggestions for psychological counseling}

Considering that IMDs children stay at home for a long time, with small space for activities, they may have long-term depressed mood, poor appetite and other manifestations, the guardians should use a variety of parentchild activities for interaction and regulation, such as poetry reading, painting, calligraphy, radio gymnastics, shuttle kicking, rope skipping and other sports.

Therefore, family members need to weigh the pros and cons fully, evaluate rationally, and well protected. We emphasize that although most short-term delayed reviews may not have a significant impact on children's health, this does not mean that no review is needed. However, review should be conducted immediately after the end of COVID-2019 epidemic, to improve disease control and reduce the risk of disability and teratogenesis of IMDs. Most of our recommendations are based on Chinese current status of medical system. The contingency suggestion will be continuously modified, which should be updated based on the ability of diagnosis and treatment in district hospital. Medical service providers should also continually update their knowledge and skills on prevention and control of the COVID-2019.

\section{Acknowledgments}

Funding: This work was partly financial supported by Chongqing Science and Technology Bureau (cstc2019jscxmsxm0189). The funders had no role in study design, data collection and analysis, decision to publish, or preparation of the manuscript. The fund was supported by Lin Zou.

\section{Footnote}

Conflicts of Interest: All authors have completed the ICMJE uniform disclosure form (available at http://dx.doi. org/10.21037/atm.2020.03.60). The authors have no conflicts of interest to declare.

Ethical Statement: The authors are accountable for all aspects of the work in ensuring that questions related to the accuracy or integrity of any part of the work are appropriately investigated and resolved.

Open Access Statement: This is an Open Access article distributed in accordance with the Creative Commons Attribution-NonCommercial-NoDerivs 4.0 International License (CC BY-NC-ND 4.0), which permits the noncommercial replication and distribution of the article with the strict proviso that no changes or edits are made and the original work is properly cited (including links to both the formal publication through the relevant DOI and the license). See: https://creativecommons.org/licenses/by-nc-nd/4.0/.

\section{References}

1. Li Q, Guan X, Wu P, et al. Early Transmission Dynamics in Wuhan, China, of Novel Coronavirus-Infected Pneumonia. N Engl J Med 2020;382:1199-207.

2. Chen N, Zhou M, Dong X, et al. Epidemiological and clinical characteristics of 99 cases of 2019 novel coronavirus pneumonia in Wuhan, China: a descriptive study. Lancet 2020;395:507-13.

3. Chen ZM, Fu JF, Shu Q, et al. Diagnosis and treatment recommendations for pediatric respiratory infection caused 
by the 2019 novel coronavirus. World J Pediatr 2020.

[Epub ahead of print].

4. Wang J, Qi H, Bao L, et al. A contingency plan for the management of the 2019 novel coronavirus outbreak in neonatal intensive care units. Lancet Child Adolesc Health 2020;4:258-9.

5. Wang W, Zou L, Wang Z. Newborn screening and prenatal diagnosis laboratory management, 1st ed.; People's Health Publishing House; Beijing, China, 2018:1-3.

6. The prevention and control law of infectious diseases of the People's Republic of China. Available online: https:// baike.so.com/doc/5412518-5650655.html

7. Tong $\mathrm{Y}$, Wang $\mathrm{M}, \mathrm{Xu} \mathrm{W}$, et al. Proposal for detection of 2019-nCoV nucleic acid in clinical laboratories. Chn J Lab Med 2020;43:E003.

8. Wang C. Biosafety Consensus for 2019 Novel Coronavirus Pneumonia in clinical laboratory. Chn J Lab Med 2020;43:E001.

Cite this article as: Liu S, Wang D, Wan K, Liu H, Zhang D, Yang J, Yuan Z, He X, Wang J, Ou M, Zhu W, Qu Y, Zhao D, Wang W, Liu S, Gu M, Huang S, Wang Z, Zou L. Chinese expert brief consensus on newborn screening of inherited metabolic disorders during the novel coronavirus infection epidemic. Ann Transl Med 2020;8(7):429. doi: 10.21037/ atm.2020.03.60
9. WHO. Laboratory testing for 2019 novel coronavirus (2019-nCoV) in suspected human cases. Available online: https://www.renrendoc.com/p-49426389.html

10. van Spronsen FJ, van Wegberg AM, Ahring K, et al. Key European guidelines for the diagnosis and management of patients with phenylketonuria. Lancet Diabetes Endocrinol 2017;5:743-56.

11. National Health Commission of the People's Republic of China. Management plan for the persons suspicious exposing or close contacting the patients with Novel coronavirus pneumonia. Available online: http://www.nhc. gov.cn/xcs/zhengcwj/202001/c67cfe29ecf1470e8c7fc47d3b 751e88.shtml

12. National Health Commission of the People's Republic of China. Novel coronavirus transmission routes and prevention guidelines. Available online: http://www.nhc. gov.cn/jkj/s3578/202001/9e73060017d744aeafff8834fc038 9f4.shtml 